\title{
Small is different: energetic, structural, thermal, and mechanical properties of passivated nanocluster assemblies
}

\author{
Uzi Landman and W. D. Luedtke \\ School of Physics, Georgia Institute of Technology, Atlanta, GA 30332-0430, USA \\ Received 9th October 2003, Accepted 9th October 2003 \\ First published as an Advance Article on the web 23rd October 2003
}

\begin{abstract}
We explore, with the use of extensive molecular dynamics simulations, several principal issues pertaining to the energetics of formation of superlattices made through the assembly of passivated nanoclusters, the interactions that underlie the cohesion of such superlattices, and the unique mechanical, thermal and structural properties that they exhibit. Our investigations focus on assemblies made of crystalline gold nanoclusters of variable sizes, passivated by monolayers of alkylthiol molecules. An analytic optimal packing model that correlates in a unified manner several structural characteristics of three-dimensional superlattice assemblies is developed. The model successfully organizes and systematizes a large amount of experimental and simulation data, and it predicts the phase-boundary between different superlattice structural motifs that evolve as a function of the ratio between the chain-length of the extended passivating molecules and the radius of the underlying gold nanocluster. The entropic contribution to the formation free energy of the superlattice assembly is found to be large and of similar magnitude as the potential energy component of the free energy. The major contribution to the cohesive potential energy of the superlattice is shown to originate from van der Waals interactions between molecules that passivate neighboring nanoclusters. The unique mechanical, thermal, thermomechanical, and thermostructural properties of passivated nanocluster assemblies, are discussed.
\end{abstract}

\section{Introduction}

Characterization and elucidation of size-evolutionary patterns of the properties of finite materials aggregates exhibiting discrete quantized energy level spectra and specific structures and morphologies, investigations of unique properties of finite-size materials clusters, and studies of the nature of the evolution from the molecular and cluster regimes to the bulk phase, are among the major challenges of modern materials science, and as such these issues have been the subject of intensive research endeavors. These investigations include explorations of structural, electronic, thermodynamic, spectroscopic, and chemical properties of isolated clusters and their assemblies. Nanophase materials built through the assembly of nanometer-scale units into ordered superlattices offer exciting perspectives as novel materials whose optical, electronic, magnetic, transport, mechanical and thermodynamic properties may be controlled by the selection of the composition and sizes of the building-block units. Such diversity and tunability suggests potential utilization of these materials as components in future nanoscale-based electronic, optoelectronic, and sensor technologies. 
Investigations of such finite aggregates of limiting small sizes and reduced dimensionalities, open avenues for systematic explorations of the physical factors and unifying principles that underlie the transition from the atomic and molecular domain to the condensed phase regime. Such behavior, where the dependence of the behavior of the system on it's size is non-scalable with the physical size of the system, but rather where Small is Different in an essential way, is emergent in nature; that is, the exhibited behavior is characteristic of the assembly of particles rather than being a property of the individual constituents, showing behavior on a larger scale then that characterizing the (relatively local) interactions between the elementary components of the system. Identification and understanding of the microscopic origins of such emergent phenomena, are of fundamental importance for elucidation of the principles of self-assembly ${ }^{1-9}$ and self-selection operative at the nanoscale.

The general methodology of "nanoparticle assembly", to which the 125th Faraday Discussion is devoted, is illustrated in Fig. 1, where stages in the passivation and assembly of gold nanocrystallites into superlattices, are depicted. In particular, the displayed images of an isolated $\mathrm{Au}_{1289}$ truncated octahedral (TO) cluster, passivated by a monolayer of dodecane thiol molecules, illustrate "bundling" of the coating molecules at the lowest temperature $(100 \mathrm{~K})$, their transformation to partial intermolecular order at $300 \mathrm{~K}$, and eventual melting of the passivating monolayer at 375 $\mathrm{K}$. Moreover, the images on the right illustrate the superlattice structures formed through assembly of the individual clusters, transforming from a bec orthorhombic arrangement at $100 \mathrm{~K}$, to a bcc superlattice at $375 \mathrm{~K}$.

Preparation of such materials often requires passivation of the "building blocks" (that is, the individual clusters) to protect against modifications of their properties by their environment, as well as to inhibit their propensity to sinter. Such passivation can be achieved in various ways. One of the most elegant passivation routes is through self-assembly, that is spontaneous formation of passivating monolayers (of organic molecules such as n-alkylthiols), which has been shown to provide a viable means of controlling the physical and chemical properties of extended solid surfaces $^{10-15}$ and nanocrystalline facets. ${ }^{16-26}$

Experimental and theoretical investigations on self-assembled monolayers (SAMs), in particular n-alkylthiols chemisorbed on extended gold surfaces $(\mathrm{Au}-\mathrm{RS})$, have been performed now for a rather long time, and they have yielded a wealth of information ${ }^{10-15,27-29}$ On the other hand, research efforts aimed at understanding the adsorption of such molecules on finite crystallites (which expose various crystalline facets, edges and corner sites) and the intermolecular organization of the molecular chains on such nanocrystallites and their mechanical and thermal properties, including possible disordering and eventual melting phase transitions, ${ }^{24-26,30-32}$ are more recent. Exploration of such systems, and studies of the dependencies of their properties on the size of the underlying gold nanocrystallites and chain-length of the passivating molecules are the focus of this article.

\section{Structural, mechanical, and thermal properties of passivated nanocluster assemblies}

We focus here on the structural, mechanical and thermal properties of superlattices whose basic units are gold nanocrystals passivated by alkylthiol molecules. The principal theoretical methodology employed by us is molecular dynamics (MD) simulations (see Appendix for pertinent details), where the equations of motions of the interacting particles are solved numerically with ultra-high spatial and temporal resolution. The MD simulations are used here in various modes, including: (a) as a technique for determining optimal structures (that is, locating minima of the multidimensional potential-energy, or free-energy, hypersurface), and (b) as means for investigating the dynamic behavior of the studied systems at finite temperature, including thermal disordering and melting transitions, thermally-driven structural transformations, and finite-temperature fluctuations used for evaluation of the mechanical elastic response characteristics of nanocluster assemblies. Whenever possible we compare our simulation results with experimental data.

Part of our work involves systematic studies of superlattices that are formed by varying the passivating chain-length and the nanocrystal core size. Based on this work, a simple model is proposed that relates the superlattice structure to the extent that the passivating chains fill the 


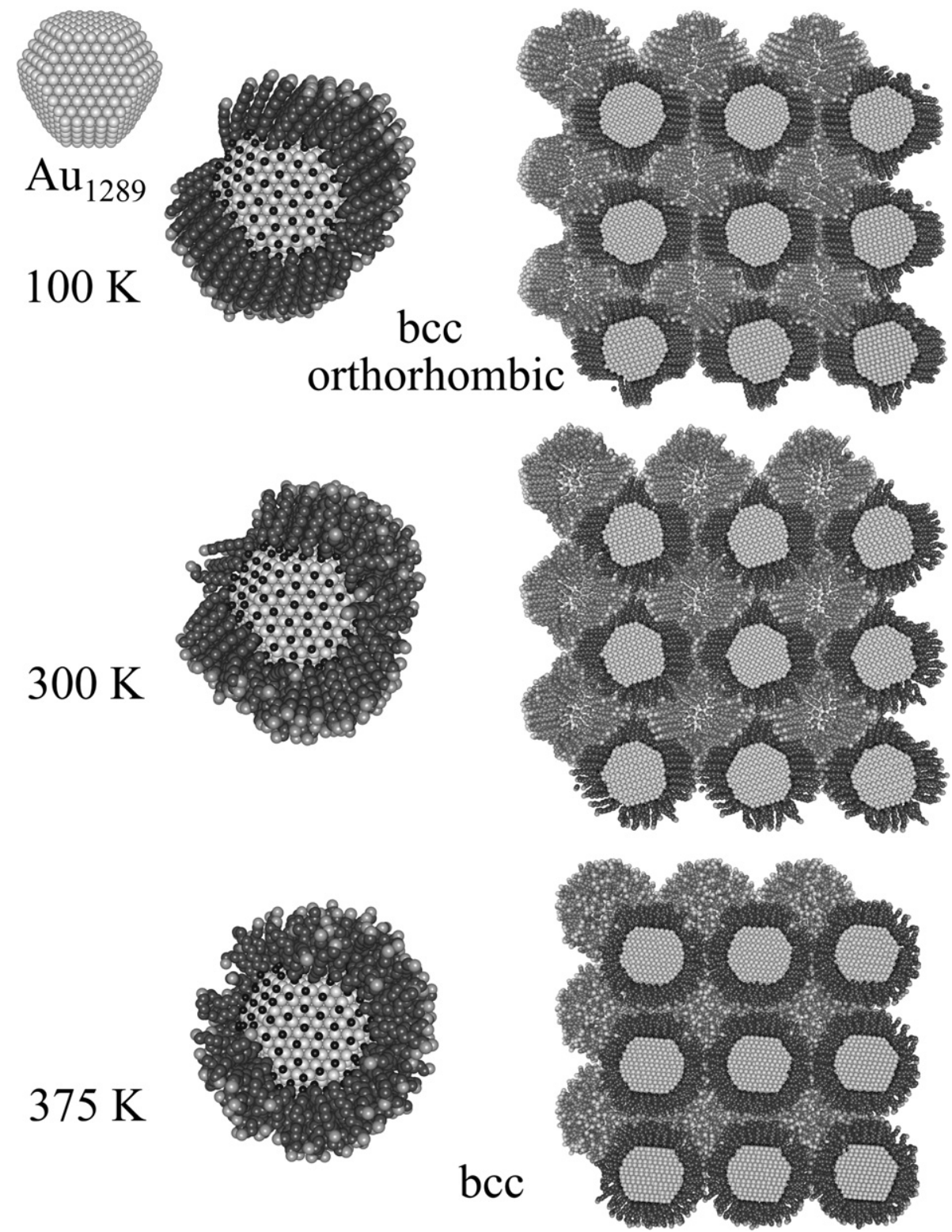

Fig. 1 Illustration of the methodology of nanoparticle assemblies, using configurations obtained through molecular dynamics simulations. (i) Top left: atomic configurations of a bare fcc crystalline gold nanocluster with a truncated octahedral morphology, containing 1289 atoms. (ii) Left column: the gold nanocluster passivated by a monolayer of dodecane thiol molecules-(top) a structure at $100 \mathrm{~K}$ exhibiting directional "bundling" of the passivating molecules; (middle) a somewhat disordered thiol layer at $300 \mathrm{~K}$; (bottom) A melted passivation monolayer at $375 \mathrm{~K}$. (iii) Right column: superlattices made through assemblies of the individual passivated nanoclusters. The structures of the superlattices exhibit a temperature dependence, with the one at $100 \mathrm{~K}$ having a bcc orthorhombic structure and the one at $375 \mathrm{~K}$ transforming to a bcc structure. Note that the passivating molecules can exhibit packing arrangements in the superlattice (right column) that are different from those found on isolated nanoclusters (left column). 
available space of the superlattice. The predictions of this model compare well with both the results of our simulations as well as with available experimental results. Other areas on which we focus relate to studies of phase transitions and structural transformations of a nanocluster superlattice induced by pressure or thermal effects, and explorations of correlations between the above properties and the manner in which the passivating hydrocarbon chains pack and arrange themselves in the superlattice for various temperatures and pressures. In addition, we explore the various contributions to the free energy (potential energy and entropic) when initially isolated passivated nanoclusters are brought together to form a superlattice.

\subsection{Nanocrystal core size, chain length of passivating molecules, chain packing, and superlattice structures}

Investigations of superlattices composed of passivated nanocrystals ${ }^{4,33}$ as well as of analogous superlattices made from larger scale micelles, ${ }^{34,35}$ have shown that the basic symmetry of the system is strongly related to the ratio, $x$, of the chain length of the passivating molecules to the cluster core radius, with systems of small $x$ displaying face-centered-cubic (fcc) superlattice symmetries and those with $x$ above a critical value displaying body-centered-cubic (bcc) symmetries. ${ }^{4,33}$ However theoretical understanding of these observations is largely lacking; in fact, using a common notion that nanoclusters protected by monolayers of disordered chain molecules pack as adhesive hard spheres, one would expect fcc packing to always dominate. By exploring through MD simulations (see below) the symmetries and properties of a range of superlattices built through the assembly of nanocrystalline cores (in the following we, will often refer to the solid crystalline nanocluster portion of a passivated gold nanocluster as a "core") containing from 140 to $1289 \mathrm{Au}$ atoms and passivated by alkylthiol molecules ranging from butane thiol $\left[\mathrm{S} \mathrm{CH}_{2}\right)_{3} \mathrm{CH}_{3}$, sometimes referred to

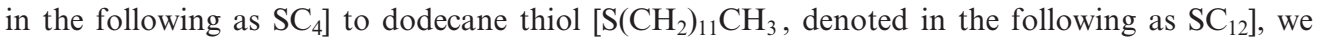
indeed observe the aforementioned structural dependence on the ratio $x$ between the chain-length and the core size. Through investigations targeted at identifying the factors that control the abovementioned dependence we find that for small $x$ the passivating chains are not long enough to completely fill the available superlattice space and fcc superlattice symmetries dominate. However, as the chain-length increases (for a given core size) there is a point at which the passivating chains anchored to a given core can extend to, and interact directly with, the chains belonging to it's second-nearest-neighbors in a bcc superlattice. It is at this point that superlattice symmetries and properties begin to display significant effects related to aspects of space filling by the hydrocarbon chains, and beyond this critical value of $x$ bcc/bct superlattice symmetries dominate (bct denotes a body-centred tetragonally distorted structure).

In light of these observations we propose a simple analytic model that incorporates insights gained from our simulations. The model involves an assumed propensity for optimization of the packing arrangement (high density) of the passivating chains in the contact region between nearestneighbor passivated clusters, in conjunction with a simple criterion that determines when packing of the chains becomes space filling; in this context we note a similar construction used earlier in studies of the two-dimensional (2D) packing and of the phases of Langmuir monolayers composed of passivated nanocrystals. ${ }^{36,37}$ In the model that we develop (referred to in the following as the "optimal packing model" or OPM) the three-dimensional packing of the passivating chain molecules is characterized as a function of the aforementioned ratio $(x)$ between the chain-length and the core radius, and it yields detailed results pertaining to the superlattice size (inter-core distances), symmetry (e.g. fcc, bcc, etc.), core volume-fraction, and overall chain free-volume. Moreover, the OPM allows prediction of the crossover point between fcc and bcc superlattice symmetries (referred to in the following as the fcc/bcc phase-boundary, $x_{\mathrm{B}}$ ). These model predictions compare well with the results of our simulation, as well as with recent experimental data.

We introduce first certain pertinent definitions and concepts (see ref. 36 for similar ideas applied for other purposes and also refs. 21 and 37 for related work). When a single nanocrystal core is fully covered by passivating alklythiol chains, the chain head group (the sulfur atom) will occupy a characteristic area, $\left(A_{0}\right.$, see Fig. 2$)$ at its contact point with the core, which determines the opening angle of a cone whose length is the core radius $(R)$ plus the length of the fully extended chain $(L$, see Fig. 2a). The volume of the conical section bounded by the area $A_{0}$ at radius $R$ and by the area $A_{l}$ at the radial distance $R+l$ from the center of the core, is denoted by $V_{l}$ (for $l=L$, this is the "excess 
(a)

(b)
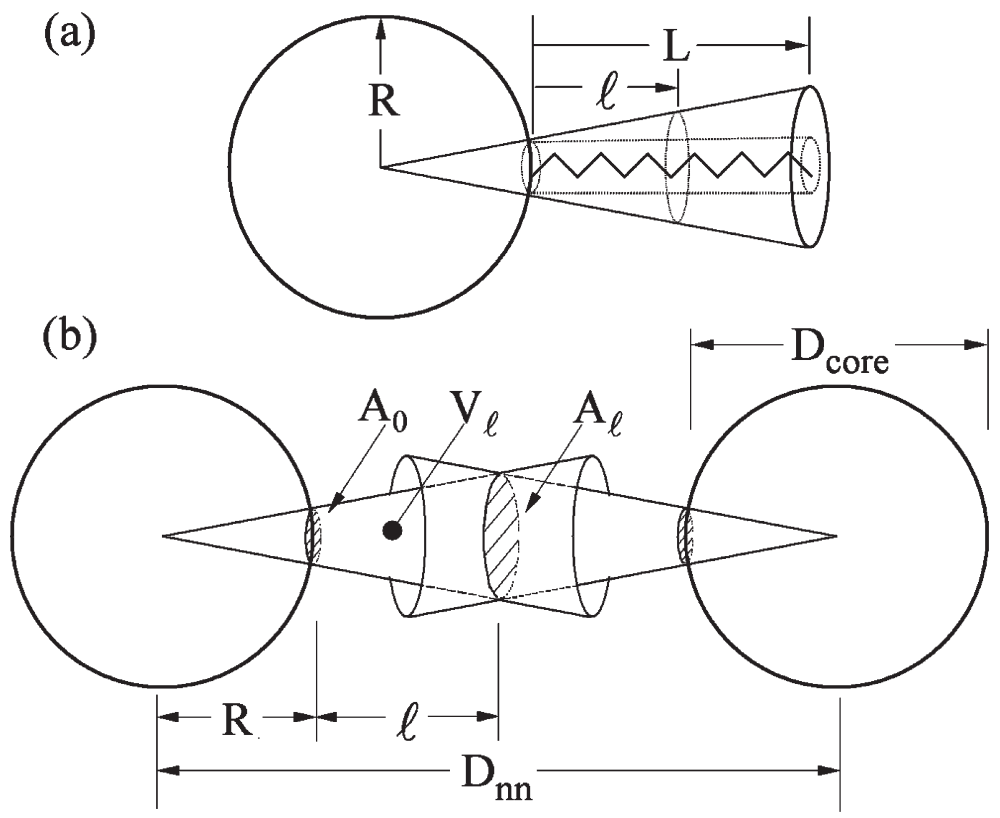

Fig. 2 Schematic illustration of quantities used in the development of the optimal packing model (OPM). (a) A single nanocluster, modeled as a sphere of core radius $R$, and an adsorbed chain molecule whose fully extended length is $L$. (b) Neighboring nanoclusters whose centers are separated a distance $D_{\text {nn }}$ apart, with overlapping passivating molecules that occupy (each) a conical section of volume $V_{l}$.

volume" 36 available to a passivant). In constructing our model we postulate that in a superlattice passivating chains between nearest-neighbor nanocrystal cores are densely (or optimally) packed, such that each chain occupies a volume $V_{l}$ with $l$ equal to half the distance between the nanocrystal cores (Fig. 2b). As a measure of alkylthiol chain packing we take as a reference volume the one defined by the base area $A_{0}$ and the length of the fully extended chain $L$, i.e. $V_{\mathrm{O}} \equiv A_{0} L$ (see Fig. 2a). The above postulate may then be represented by a simple relationship between $V_{l}$ and $V_{\mathrm{O}}$ (see below). Similar to the earlier definition of $x=L / R$ (the extended chain-length divided by the core radius), we define $x_{l}=l / R$, i.e. a distance $l$ from the core surface divided by the core radius $R$; obviously $x \equiv x_{L}$. We note that the fully extended chain-length, $L$, is a function of the number of carbons atoms, $n$, in the chain. In this study we use $L=0.12(n+1) \mathrm{nm}$ (see refs. 4 and 33).

With these definitions we find that the volume $V_{l}$ of the cone bounded by the areas $A_{0}$ and $A_{l}$ is given by

$$
V_{l}=A_{0} l\left(1+x_{l}+x_{l}^{2} / 3\right)
$$

Taking now the distance $l$ to be half the distance between two passivated nanocrystal cores when they are nearest-neighbors in a superlattice (see Fig. 2 b), i.e. $l=\left(D_{\mathrm{nn}}-D_{\text {core }}\right) / 2$, one can write a defining equation for our model,

$$
V_{l}=\lambda V_{\mathrm{O}}
$$

where, as we find below, $\lambda$ is close to unity. Namely, the passivating chains lying directly between two nearest-neighboring cores, and occupying a volume $V_{l}$ for each core (see Fig. 2b), will pack with essentially the same density as fully elongated chains confined to a cylinder of cross-sectional area $A_{0}$ and length $L$. In the case of alkylthiol chains, this is a good assumption. However, one should note that the base area $\left(A_{0}\right)$ depends on the nature of the head group (sulfur in our case) with different head groups associated with different chain packing densities. If the base area is small enough to result in a corresponding higher density, then a value of $\lambda>1$ is likely to lead to a better fit to experimental data. Through comparisons between the OPM with results of our 
MD simulations, we have concluded that this density is comparable to that of a typical hydrocarbon liquid. As we will show, taking $\lambda=1$ gives results in very close agreement with experiment. The scaling factor $\lambda$ allows one to examine and quantify how the consequences of eqn. (2) change when the packing density of the chains is varied. We note that $x$, the ratio of the fully extended chain-length to core radius $R$, will be taken in most of what follows as an independent variable used to characterize physical properties of superlattices composed of passivated nanocrystalline cores.

We may now derive a number of consequences of eqn. (2), that facilitate direct comparisons with experimental results. ${ }^{4,33}$ In particular, using the definitions of $V_{l}$ and $V_{\mathrm{O}}$ in eqn. (2) leads to the third-order equation

$$
\left(x_{l}+x_{l}^{2}+x_{l}^{3} / 3\right)=\lambda x
$$

which has one real root

$$
x_{l}=(1+3 \lambda x)^{1 / 3}-1 .
$$

Using the relation $l=\left(D_{\mathrm{nn}}-D_{\text {core }}\right) / 2$ given earlier, we have from eqn. (4)

$$
D_{\text {nn }}-D_{\text {core }}=D_{\text {core }}\left[(1+3 \lambda x)^{1 / 3}-1\right] \text {. }
$$

Before comparing our results to experimental data and MD simulation results, we discuss a further model assumption, relating to the core volume fraction and superlattice symmetry as functions of $x$. It has been observed experimentally, ${ }^{4,33}$ as well as in the present study, that fcc superlattices prevail when $x$ is smaller than $\sim 0.7$, and that above this value bcc structures dominate. We have found that a very simple assumption on the general distribution of chains in a superlattice, along with the consequences of the other main model assumption expressed in eqn. (2), provide a basis for understanding both the change in superlattice symmetry that occurs at $x \sim 0.7$, as well as a number of other related changes in properties that occur when this value of $x$ is reached. As aforementioned, fcc structures are found when the ratio of chain-length to core radius is small. In a sense, for the low $x$ regime, the passivated gold nano-crystallites close-pack somewhat like adhesive hard cores (i.e. hard "sticky" spheres), since in most of these systems the passivating chains are in a liquid-like state at $300 \mathrm{~K}$. In this regime, one would find for a bcc superlattice composed of the these low- $x$ clusters that the chains from one core could not reach, even if fully extended, the chains of a second-(next-) nearest neighbor (nnn).

The above observations lead us to conclude that the bcc superlattice first becomes stabilized and energetically favored over competing fcc structures when the chains from one solid core begin to have appreciable direct interaction with those of a nnn core in a bcc lattice. We implement this idea as follows: taking passivated cores that comprise an fcc superlattice (i.e. in the small $x$ regime) and considering a bcc superlattice composed of these same passivated cores, the phase boundary between the fcc and bcc superlattice structures is reached when the chain-ends from two nnn bcc cores begin first to touch. Relating $D_{\mathrm{nnn}}$ to $D_{\mathrm{nn}}$, the above implies that

$$
D_{\mathrm{nnn}} \equiv 2 D_{\mathrm{nn}} / 3^{1 / 2}=D_{\text {core }}+2 L \text {. }
$$

Using eqns. (5) and (6), one can show that the condition on $x \equiv x_{\mathrm{B}}$ at the fcc/bcc phase boundary, eqn. (6), may be written as

$$
\left(2 / 3^{1 / 2}\right)\left(1+3 \lambda x_{\mathrm{B}}\right)^{1 / 3}=1+x_{\mathrm{B}}
$$

This leads to a cubic equation that has three real and unequal roots, with only one of them being positive and physically meaningful. Since the exact result is fairly complicated we give below the values for $x_{\mathrm{B}}$ corresponding to some selected values of $\lambda$. For $\lambda=1$, which yields a particularly good correspondence with experimental results, one finds $x_{\mathrm{B}, \mathrm{Exp}} \cong 0.664$ that is consistent with the experimental value $e^{4,33}$ of $\sim 0.7$; chain-filling considerations have been used in refs. 4 and 33 for estimation of the phase boundary. However, when comparing OPM predictions to the results of our MD simulations, we have found that a value $\lambda=1.15$ leads to a better correspondence with the simulation data. Solving eqn. (7) for $\lambda=1.15$, one finds $x_{\mathrm{B}, \mathrm{MD}} \cong 0.790$, which is somewhat higher than the experimental result, but still in satisfactory agreement with the experimentally observed value. As noted above, the deviation of $\lambda$ from unity (i.e. $\lambda=1.15$ ) is most likely related to the 
description of the head group (sulfur) in our MD simulations, with the current choice of potential parameters resulting in a somewhat denser packing of the passivating molecules than estimated experimentally. Changing the length parameter corresponding to the sulfur head group will lead to a value of $\lambda$ closer to unity. In the following, the phase-boundary values, $x_{\mathrm{B}}$, used in discussing the experimental and MD results are denoted by $x_{\mathrm{B}, \mathrm{Exp}}$ and $x_{\mathrm{B}, \mathrm{MD}}$, respectively.

We now turn to considerations pertaining to the solid nanocrystalline core-volume fraction in a passivated cluster superlattice, which is found by dividing the total core volume in a unit cell by the total volume of the unit cell. For a fcc lattice, $x<x_{\mathrm{B}}$, the core volume-fraction can be written as $V_{\text {fcc }}=\pi /(3 \sqrt{ } 2)\left(D_{\text {core }} / D_{\mathrm{nn}}\right)^{3}$, yielding with the use of eqn. (5)

$$
V_{\mathrm{fcc}}=\pi /(3 \sqrt{ } 2) /(1+3 \lambda x) \text {. }
$$

The numerator $\pi /(3 \sqrt{ } 2) \cong 0.7405$ is the volume fraction for a hard-sphere fcc lattice, obtained when $x=0$ and the nearest-neighbor cores directly touch.

For a bcc lattice, (i.e., when $\left.x>x_{\mathrm{B}}\right)$, the core volume fraction can be written as $V_{\mathrm{bcc}}=\pi(\sqrt{ } 3 / 8)$ $\left(D_{\text {core }} / D_{\mathrm{nn}}\right)^{3}$, which can be written as

$$
V_{\mathrm{bcc}}=\pi(\sqrt{ } 3 / 8) /(1+3 \lambda x) .
$$

The numerator $\pi \sqrt{ } 3 / 8 \cong 0.6802$ is the volume fraction for a hard sphere bcc lattice.

We are now in a position to present and compare predictions of the OPM with experimental findings (in direct comparisons of model predictions to experimental results we use $\lambda=1.0$ in the model equations), and with results of our MD simulations (for a brief description of the MD simulation methodology see the Appendix). In Fig. 3 we display as a function of $x$, OPM

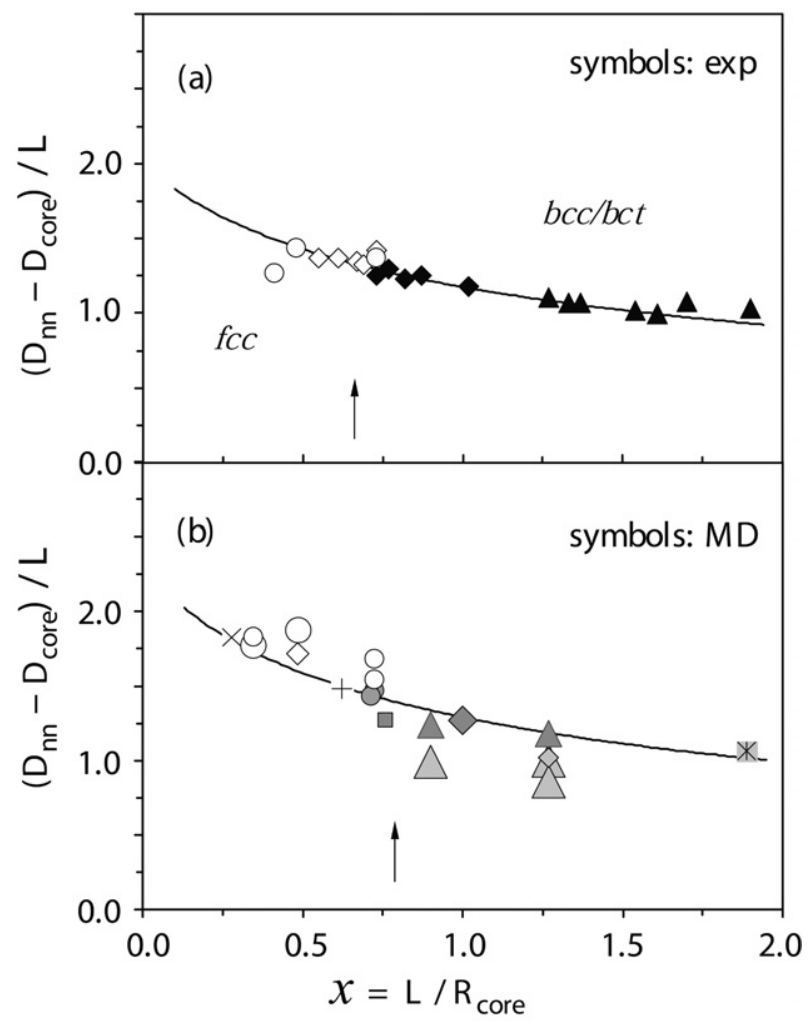

Fig. $3\left(D_{\text {nn }}-D_{\text {core }}\right) / L$ plotted versus the parameter $\chi=L / R_{\text {core }}$. In (a) the symbols represent experimental data and in (b) they represent results from molecular dynamics simulations. Open and filled symbols represent fcc and bcc/bct superlattices, respectively. The various symbols correspond to systems specified in Fig. 5. The lines in (a) and (b) depict predictions of the optimal packing model (OPM). 
predictions (eqn. (5), solid lines) as well as experimental data ${ }^{33}$ (in Fig. 3a) and MD simulation results (in Fig. 3b) for the distance between the surfaces of nearest-neighbor cores (in units of the fully extended chain length $L$ ) which provides a measure of the overlap between chains from nearestneighbor cores. It is evident from the figure that the predictions of the OPM agree rather well with both the experimental and MD simulation data. For chain lengths that are very small compared to the core size (small $x$ values) the inter-core surface distances approach $2 L$ (as $x$ approaches zero), and there is virtually no chain overlap: in this regime the passivated cores are arranged in open structures, such as fcc superlattices. For relatively large chain lengths (large $x$ ) the overlap between chains grows to the point that when $x \sim 2$ the separation between the surfaces of two neighboring gold cores is only about one chain-length $(L)$. This distance is predicted to decrease even more as the ratio between the chain-length and the core size further increases. It should be noted that this decrease in nearest-neighbor surface separation (in units of $L$ ) is likely to be related to several factors, including: an increased interpenetration of disordered inter-core chains, a large amount of chain packing into (and filling-up of) the available open space away from the region directly between nearest-neighbor cores, and a propensity for formation of chain "bundles" (that have a tendency to form when chain-ordering occurs) which may be oriented away from the nearestneighbor inter-core space, thereby allowing a smaller core-core separation.

Another point of comparison is the core (here, gold nanocluster) volume fraction, $V_{\mathrm{Au}}$. The experimental data is presented in Fig. 4. Once $x_{\mathrm{B}}$ is determined, then the core volume fraction for the fcc structures (eqn. (8a)) is used for $x<x_{\mathrm{B}}$, while eqn. (8b) is used for $x>x_{\mathrm{B}}$ where bcc structures are predicted to prevail. Using eqns. (8a) and (8b), with $x_{\mathrm{B}}=x_{\mathrm{B} \text {,Exp }}$ given above, leads to the solid curve in Fig. 4 where once again a very good agreement with the experimental results is observed.

Before comparing OPM predictions to the MD results, we comment on certain pertinent characteristics of the systems that were simulated by us. First we note that these systems were selected such that they will be representative of various characteristic variables, such as: the gold nanocrystalline core size and crystallography, the superlattice temperature and symmetry, the chain-length of the passivating molecules, and the phase behavior (solid-like or liquid-like) of the passivating chains. Additionally, we remark that the phase behavior of the passivating chains encompasses two states. ${ }^{30,31}$ One is a disordered liquid-like (or glassy) state where interdigitation of inter-core chains may occur; most of the systems simulated by us belong to this class. The other state of the superlattice is a more ordered one (as measured by high intra-chain trans configuration values) where direct inter-core chain interdigitation on the single chain level is not possible: rather, a higher degree of inter-chain alignment occurs, i.e., formation of multi-chain "bundles" that may introduce variations or disruptions in the superlattice symmetry as the system attempts to lower its energy through the arrangement of such bundles.

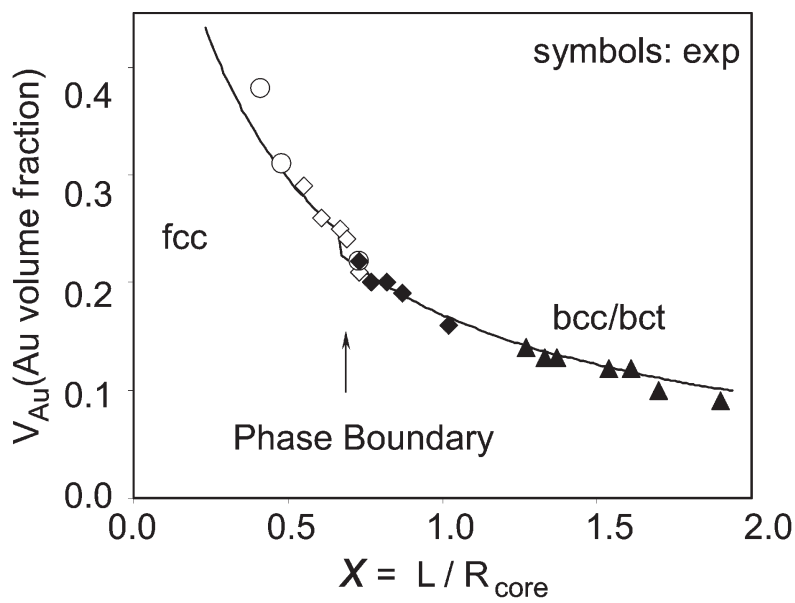

Fig. 4 Gold nanocluster volume fractions in superlattices plotted versus the parameter $\chi$. The symbols depict experimental data, and the systems that they represent are specified in Fig. 5. Open and filled symbols represent fcc and bcc/bct superlattices, respectively. The line depicts predictions of the optimal packing model (OPM). 
Core volume-fractions, $V_{\mathrm{Au}}$, are plotted versus $x$ in Fig. 5 for the MD simulations (symbols) as well as for the OPM (solid line). The large variety of systems studied here are listed in the figures. We note that systems with passivated cores of different sizes and molecular chains of different lengths, may share similar values of the chain-length to core-radius ratio $x$. We also remark that a number of systems were simulated at elevated temperatures and that some involve passivating chains with a higher degree of inter-chain order (i.e. the aforementioned bundling). The agreement between the MD results and the OPM predictions is remarkably satisfactory, particularly in light of the wide range of systems that have been simulated. As in the case of the experimental results shown in Fig. 4, systems with $x<x_{\mathrm{B}}$ exhibit close-packed fcc/hcp structures while for $x>x_{\mathrm{B}}$ more open $\mathrm{bcc} / \mathrm{bct}$ structures dominate. In this context we observe that examination of the percent

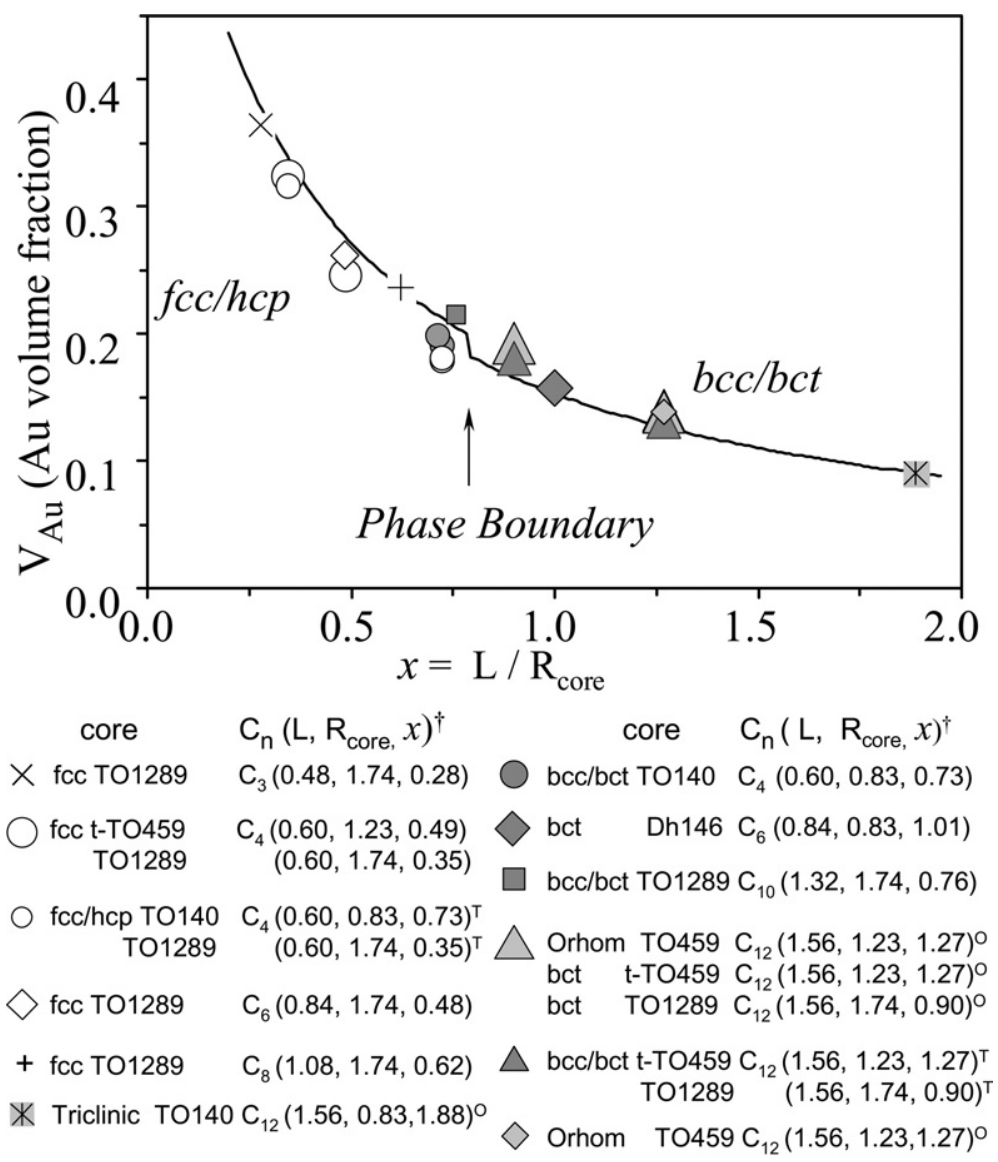

${ }^{\dagger} \mathrm{T}=300 \mathrm{~K}$ unless noted otherwise; length in $\mathrm{nm}$

${ }^{\mathrm{T}} \mathrm{T}=350-375 \mathrm{~K},{ }^{\mathrm{O}}$ High degree of chain order

Fig. 5 Gold nanocluster volume fractions in superlattices plotted versus the parameter $\chi$. The symbols depict results from molecular dynamics simulations, and the systems that they represent are specified in the bottom part of the figure. Open and filled symbols represent fcc and bcc/bct superlattices, respectively. The line depicts predictions of the optimal packing model (OPM). The first entry at the bottom gives the structure of the superlattice (i.e., fcc, triclinic, bcc, etc.); bct stands for bcc tetragonal, and Orhom stands for orthorhombic. The entry "core" describes the gold nanocluster: for example TO1289 corresponds to truncated octahedral morphology of a nanocluster containing 1289 atoms. t-TO stands for twinned TO, and Dh stands for decahedral symmetry. The entry $C_{\mathrm{n}}\left(L, R_{\text {core }}, \chi\right)^{y}$ gives the following information: $C_{\mathrm{n}}$ gives the number of carbon atoms in the passivating alkylthiol chain; $\mathrm{L}$ is the length of the fully extended chain (in $\mathrm{nm}$ ); $R_{\text {core }}$ gives the radius of the gold nanocrystalline core (in $\mathrm{nm}$ ). The meaning of the superscript $y$ is given at the bottom. 
free-volume as a function of $x$ reveals a pronounced decrease for $x>x_{\mathrm{B}}$, with the free volume of the more open structures that develop for $x>x_{\mathrm{B}}$ being similar to that of liquid dodecane (simulated by us at $300 \mathrm{~K}$ with the same interaction potentials employed in the superlattice studies). Furthermore, we note that MD simulations at $300 \mathrm{~K}$ of self-assembled monolayers of $\mathrm{SC}_{12}$ adsorbed onto an $\mathrm{Au}(111)$ surface $^{38}$ yielded smaller free-volumes then those found in our current study, indicating that while the passivating alkylthiol chains pack in a superlattice with noticeably higher density for $x>x_{\mathrm{B}}$, they do not reach the higher density occurring in SAMS adsorbed on extended gold surfaces.

Before we continue, we wish to comment on certain analogies between the phase-behavior described above as a function of $x$ (see Figs. 4 and 5) and experimental investigations of micellar suspensions. ${ }^{34,35}$ In these studies it has been found that when the ratio (denoted as $x_{\mathrm{m}}$ ) between the coronal layer thickness and the core radius is below a certain threshold value defining a phase boundary, $x_{\mathrm{mb}}$, the ordered structures of the spherical micelles will favor fcc lattices, while when $x_{\mathrm{m}}$ exceeds $x_{\mathrm{mb}}$ the ordered structures favor a bcc motif. The above is similar to the behavior found by us for passivated nanocrystalline assemblies. We note, however, that while for the passivated cores of the present study the ratio $x$ involves the fully extended chain length (on the order of a $\mathrm{nm}$ ), in the micelle case the coronal layer thickness (on the order of 10-20 nm) involves long folded polymers.

To end our discussion of chain-packing in passivated nanocluster assemblies we comment on the cohesive energies of the superlattice defined as

$$
E_{\text {cohesive }}=\left(N_{\text {cores }} E_{\mathrm{FC}}-E_{\mathrm{SL}}\right) / N_{\text {cores }},
$$

where $N_{\text {cores }}$ is the number of passivated cores in the computational unit cell of the superlattice, $E_{\mathrm{FC}}$ is the total energy of a free passivated core at the same temperature as the superlattice, and $E_{\mathrm{SL}}$ is the total energy of the superlattice system. We have found that the cohesive energies vary approximately linearly with the passivating alkylthiol chain-length (for chains containing more then four carbons), and they can reach rather high values ${ }^{30}$ (e.g. about $6 \mathrm{eV}$ for a superlattice with $x=0.28$ for $\mathrm{SC}_{4}$ passivants, and $26 \mathrm{eV}$ for $x=0.76$ for $\mathrm{SC}_{10}$ passivants). Furthermore, we found that the normalized cohesive energy $E_{\text {cohesive }}^{\prime}$ defined as $E_{\text {cohesive }}^{\prime}=E_{\text {cohesive }} /\left(-E_{\text {intercluster }}\right)$, where $E_{\text {intercluster }}$ is the interaction energy (divided by $N_{\text {cores }}$ ) between alkylthiols that belong to different cores, is close to unity for all $x$. This leads us to conclude that the cohesive energy is dominated by the van der Waals chain-chain interactions between molecules passivating neighboring gold cores, i.e. $E_{\text {intercluster }}$. We have found this to be true whenever the passivating chains are in a disordered or somewhat ordered state near $300 \mathrm{~K}$. However, for much lower temperatures where chain ordering effects occur, deviations from this behavior may be found.

\subsection{Elastic properties of nanocluster superlattices}

The special nature of the superlattices formed through assembly of passivated solid nanocrystallites (gold nanoclusters in this study), where the space between the solid cores is largely occupied by a relatively soft (and compliant) organic material, lead us to anticipate interesting unique consequences pertaining to the mechanical and thermal properties of these materials. Moreover, we expect that the mechanical properties will exhibit a dependence on the characteristic parameter $x$ introduced in the previous section.

We explored the above issues with the use of a strain fluctuation method ${ }^{39-43}$ that utilizes the dynamic treatment of the computational cell (where both the lengths of the three vectors defining the computational cell as well as the angles between them are treated as dynamical variables), and allows evaluation of the elastic constants through analysis of the MD simulation data. As aforementioned, the majority, but not all, of the systems that we have simulated have at $300 \mathrm{~K}$ passivating chains in a disordered state. These systems generally have orthogonal unit cell axes with two that are close in length (sometimes we will refer to these as axes 1 and 2) and a third one (the " 3 " axis) that may be unequal to the other ones.

The compressibility $(X)$ of the systems that we studied is shown in Fig. 6, and components of the shear modulus are displayed in Fig. 7. Also shown is the compressibility of a pure dodecane liquid for which we determined from separate simulations that $X=0.98(\mathrm{GPa})^{-1}$ at $300 \mathrm{~K}$ (the experimental $^{44}$ value is 0.99$)$. Certain trends are readily observed as the chain-length to core-radius ratio 


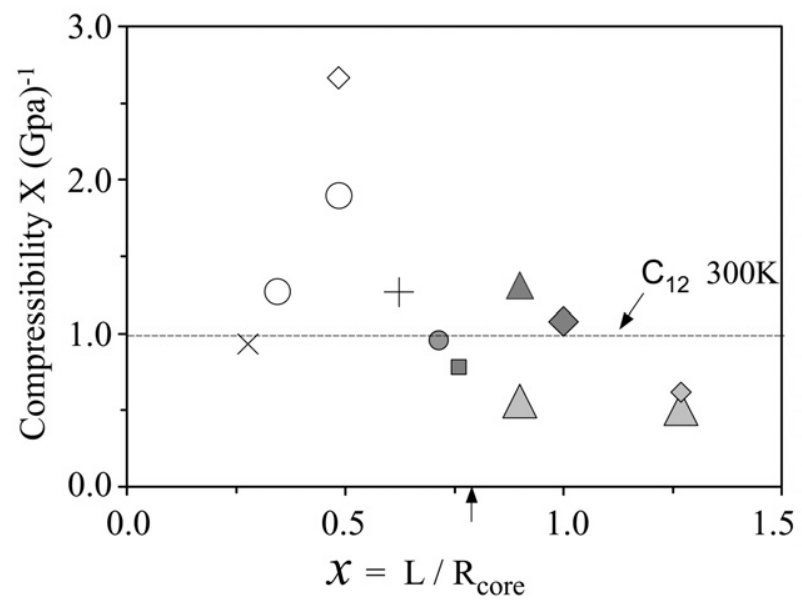

Fig. 6 The compressibility $X$ of the simulated superlattice assemblies made from the passivated gold nanoclusters, plotted versus the parameter $\chi$. Open and filled symbols represent fcc and bcc/bct superlattices, respectively. The symbols depict results from molecular dynamics simulations, and the systems that they represent are specified in Fig. 5. The value of $\mathrm{X}=0.98(\mathrm{GPa})^{-1}$ corresponds to a simulated liquid dodecane at $300 \mathrm{~K}$. The arrow at the bottom points to the value of $\chi_{\mathrm{B}}$, denoting the OPM predicted boundary between fcc and bcc/bct superlattice structures.

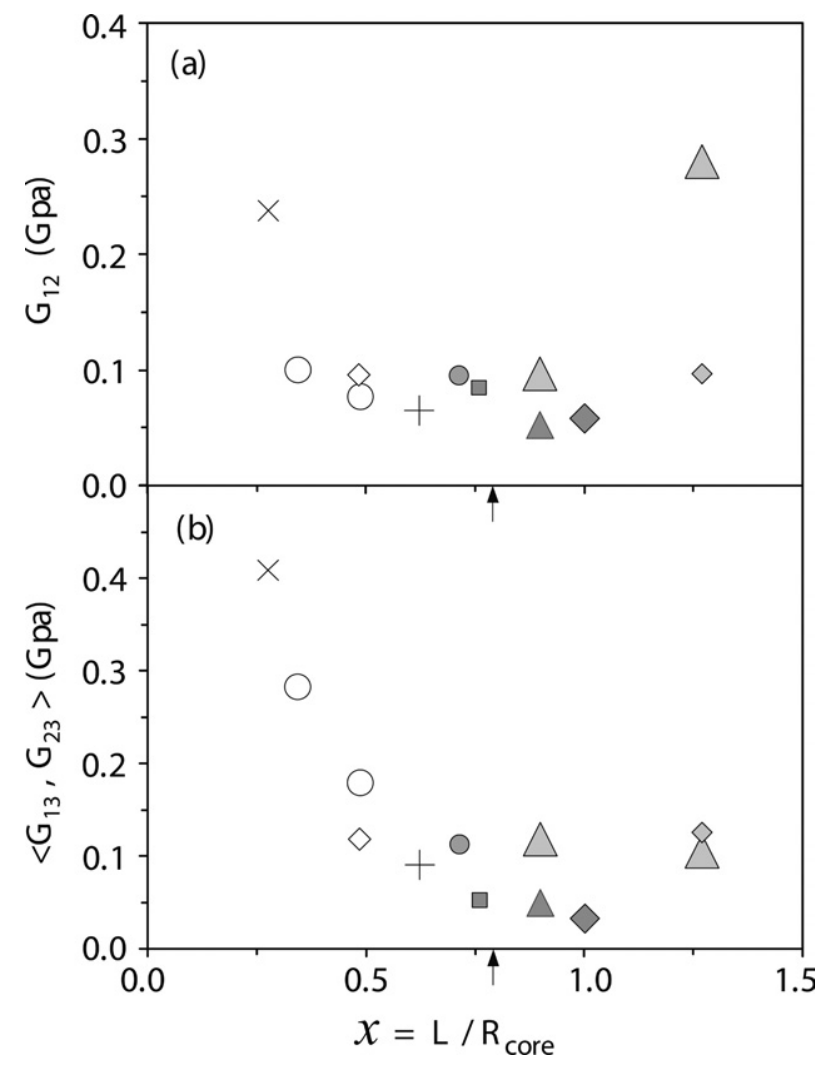

Fig. 7 (a) The shear modulus $G_{12}$ in a plane normal to the axis marked 3, and (b) the average, of the shear moduli $G_{13}$ and $G_{23}$, plotted versus the parameter $\chi$. The results are taken from MD simulations of superlattice assemblies made from passivated gold nanoclusters. For the meaning of the symbols see caption to Fig. 6, and Fig. 5. 
$x$ becomes very small, as well as when it approaches and exceeds the phase boundary at $x_{\mathrm{B}}$. For relatively short chains compared to the core radius (small values of $x$ ), the compressibility heads towards smaller values because the passivated cores are approaching the adhesive hardsphere limit. As $x$ increases, the increased relative chain-length allows the passivating molecules to not only have greater propensity for interpenetration, but also to have a larger capacity to be pushed (or squeezed) into surrounding vacant space in the superlattice.

As noted in the last section, when $x$ approaches $x_{\mathrm{B}}$, the available free-volume in the superlattice decreases dramatically leading to less space into which the chains may be pushed by the superlattice compression, and the free volumes approach values that are commonly found for hydrocarbon liquids. This behavior is reflected in the dependence of the compressibility on $x$, where upon filling-up of the available free-volume by the disordered chains $\mathrm{X}$ takes values typical of hydrocarbon liquids at room temperature. The systems that have particularly low compressibility for large $x$ are those in which the chains have achieved a higher degree of order (through the aforementioned chain-bundling); we have observed that these systems often display anomalous behavior.

The shear modulus $G_{12}$ in a plane normal to the 3-axis (Fig. 7a), and the average, $\left\langle G_{13}, G_{23}\right\rangle$, of the shear moduli $G_{13}$ and $G_{23}$ (Fig. 7b) show that both $G_{12}$ and $\left\langle G_{13}, G_{23}\right\rangle$ approach larger values for small values of $x$, with $G_{12}$ being slightly smaller than $\left\langle G_{13}, G_{23}\right\rangle$. However when $x$ approaches and exceeds $x_{\mathrm{B}}$ both moduli decrease significantly, attaining values of the order of $50 \mathrm{MPa}$ (similar to values found for elastomers) at $x_{\mathrm{B}}$ (excluding the systems with ordered chains, see in particular Fig. 7a for $x=1.27$ ).

\subsection{Thermal behavior and phase transitions}

In this section we focus on the thermodynamic and thermostructural transformations of nanocluster superlattices, with the temperature serving as the control parameter. In earlier work $^{30,31}$ we have found that when the temperature of a passivated cluster (when isolated in vacuum, or when in a superlattice) is below the freezing point of the passivating chains, they order and this has a pronounced effect on the way in which the chains are oriented and distributed, and on the symmetries of a superlattice to which they may belong. To explore these issues further we have equilibrated a superlattice composed of gold TO1289/SC 12 units (i.e., solid fcc crystalline nano-clusters containing 1289 gold atoms with a truncated-octahedral, TO, morphology, and passivated by dodecane thiol molecules) at a range of temperatures (from a very low, $1 \mathrm{~K}$, to a high one, $500 \mathrm{~K}$ ) in order to generate a caloric curve (total energy as a function of temperature) for the superlattice system. Additionally, at each temperature we computed, using the aforementioned strain fluctuation formalism, a complete set of elastic constants.

In Fig. 8 (middle curve, filled dots) we show the total energy of the superlattice system normalized by dividing by the total number of passivating alkylthiol chains. As a point of reference we also show (open dots) the caloric curve of a free passivated $\mathrm{Au} \mathrm{TO} 1289 / \mathrm{SC}_{12}$ cluster identical to the ones used for the superlattice studies. The curves that are shown are obtained by a nonlinear least-squares fit to the MD results, of the form

$$
E(T)=\varepsilon+c\left(T-T_{0}\right)+\left\{\delta \varepsilon+\delta c\left(T-T_{0}\right)\right\} \tanh \left(\left(T-T_{0}\right) / \sigma\right),
$$

with the set $\left(\varepsilon, c, \delta \varepsilon, \delta c, \sigma, T_{0}\right)$ as fitting parameters. The tanh term takes values from -1 to +1 as $T$ crosses from below the transition temperature $T_{0}$, with the transition width controlled by $\sigma$. This provides a smooth transition between the low and high temperature linear portions. When the low and high temperature linear asymptotes are extended to $T=T_{0}$, as shown in the shifted curves in Fig. 8 (for ease of display), the vertical distance between them is taken as a measure of the latent heat, given by $L=2 \delta \varepsilon$. The parameter $T_{0}$ is taken as the phase transition temperature.

The free cluster and the superlattice clusters have in common that the phases below and above the transition temperature $T_{0}$ are associated with ordered and disordered passivating alkylthiold chains, respectively. It is interesting to note that the caloric curves of both systems are quite similar. The transition temperature and latent heat of the superlattice system are found to be $T_{\mathrm{SL}}=312 \mathrm{~K}$ and $L_{\mathrm{SL}}=0.19 \mathrm{eV}\left(\mathrm{SC}_{12}\right)^{-1}$ (per alkylthiol chain) while those of the free cluster are $\mathrm{T}_{\mathrm{FC}}=292 \mathrm{~K}$ and $\mathrm{L}_{\mathrm{FC}}=0.22 \mathrm{eV}\left(\mathrm{SC}_{12}\right)^{-1}$; the full set of fitting parameters $\left(\varepsilon, c, \delta \varepsilon, \delta c, \sigma, T_{0}\right)$ that we determined for 


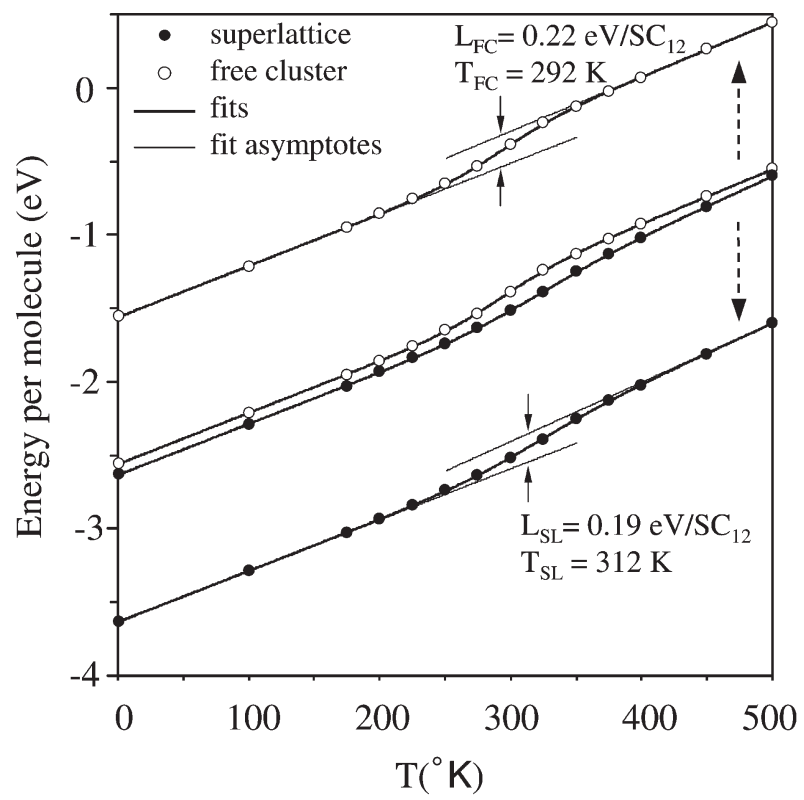

Fig. 8 Caloric curves (total energy versus temperature). Open dots correspond to results obtained from simulations of an isolated gold nanocluster containing 1289 atoms in a truncated octahedral morphology and passivated by a dodecane-thiol monolayer $\left(\mathrm{TO} 1289 / \mathrm{SC}_{12}\right)$. The filled dots correspond to the simulated superlattices made through assembly of the above gold nanoclusters. $L_{\mathrm{FC}}$ and $T_{\mathrm{FC}}$ give the latent heat and melting temperature for the free clusters, and the quantities with the subscript "SL" correspond to the superlattice assembly. The upper and lower curves are displaced replicas of the corresponding middle curves, shown for clarity. The solid lines represent line-fits using eqn. (10) (see text). The melting transitions described here correspond to phase transitions of the passivating dodecane-thiol monolayer.

the superlattice is $\left(\varepsilon=-1.45 \mathrm{eV}, c=3.75 \mathrm{meV} \mathrm{K}^{-1}, \delta \varepsilon=96.1 \mathrm{meV}, \delta c=0.28 \mathrm{meV} \mathrm{K}^{-1}, \sigma=66.4\right.$ $\left.\mathrm{K}, T_{0}=312 \mathrm{~K}\right)$, and that for the free cluster is $\left(\varepsilon=-1.43 \mathrm{eV}, c=3.60 \mathrm{meV} \mathrm{K}^{-1}, \delta \varepsilon=108.0 \mathrm{meV}\right.$, $\left.\delta c=0.11 \mathrm{meV} \mathrm{K}^{-1}, \sigma=47.5 \mathrm{~K}, T_{0}=292 \mathrm{~K}\right)$. The superlattice latent heat may be translated to more conventional units by multiplying $L_{\mathrm{SL}}$ by the number of passivating chains per cluster and dividing by the mass of the fully passivated core (including the core mass). With the above we find $L_{\mathrm{SL}}=15.5 \mathrm{~J} \mathrm{~g}^{-1}$ which is in line with experimental values for superlattices composed of $\mathrm{Ag}$ cores passivated by octadecanethiol molecules. ${ }^{45-47}$

An important measure of order in the passivating alkylthiols is the percent of trans configurations in the hydrocarbon chains; this measure has been found quite useful in earlier studies. ${ }^{30,31}$ In Fig. 9a we display for the superlattice system the percent chains with a trans configuration as a function of temperature. Additionally, we show the percentage of chains with a trans configuration for a free passivated cluster (from the same study as that presented in Fig. 8), and also for a simulated self-assembled monolayer of $\mathrm{SC}_{12}$ adsorbed onto an $\mathrm{Au}(111)$ substrate at a range of temperatures. ${ }^{38}$ Not surprisingly, both the superlattice and free-cluster chains do not reach the degree of order present in those of the SAM. However, the degree of order in the free clusters and in the superlattice are comparable. The free-cluster chains can achieve somewhat higher order than those of the superlattice at temperatures below the phase transition temperature, $T_{0}$, because of the additional packing constraints present in the superlattice.

To display more clearly the structural features of the superlattice as a function of temperature, we show in Fig. 9b the length of the three axes defining the computational unit cell. We find that the three cell axes remain orthogonal throughout the study. The " 3 " axis is taken as the inequivalent axis, as the other two are more similar in length as compared to it. It is rather evident that in the midpoint of the phase transition, i.e. for $T_{0}=312 \mathrm{~K}$, the superlattice is quite tetragonally distorted. At very low temperature the symmetry of the superlattice is orthorhombic; open structures of higher symmetry (such as bcc,bct, and fcc) emerge at higher temperatures (see Fig. 1), 


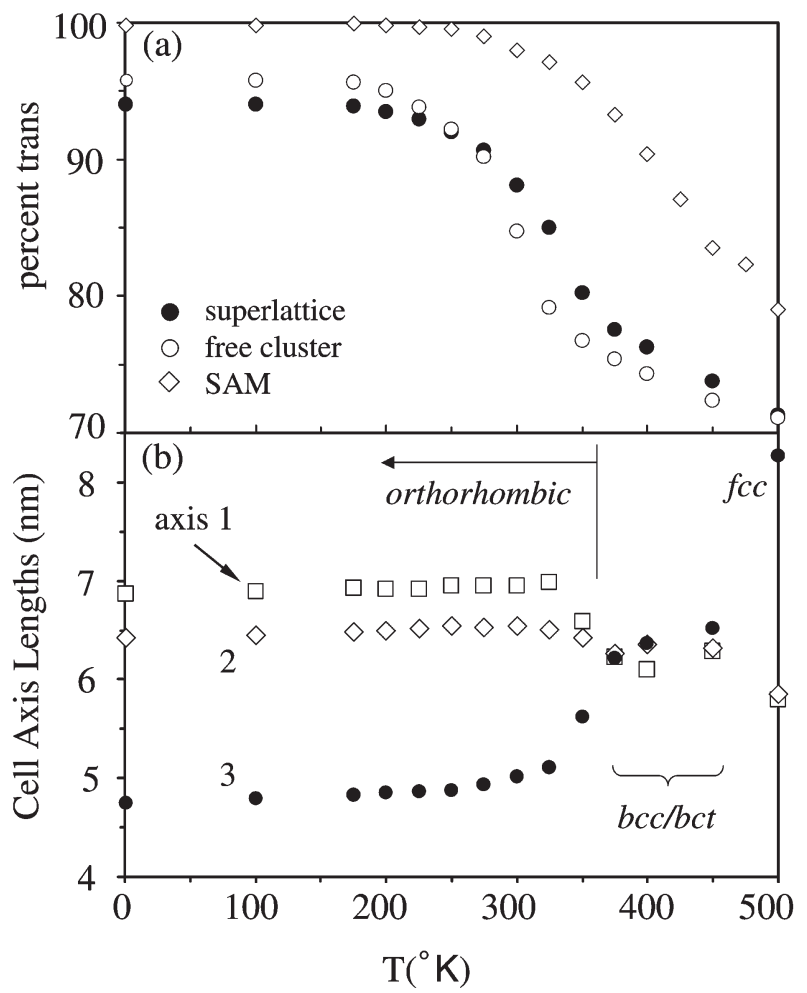

Fig. 9 (a) Percentage of trans configurations, and (b) lengths of the axes of the superlattice unit cell plotted versus temperature. The results were obtained from molecular dynamics simulations of the free cluster and superlattices described in Fig. 8. Shown in (a) are results for the free cluster and for the superlattice assembly, as well as for a simulated self-assembled monolayer (SAM) adsorbed onto an extended Au(111) surface.

and their occurrence is facilitated by the thermally disordered and mobile character of the free tails of the alkylthiol chains.

We used the strain fluctuation formalism ${ }^{39,40,42,43}$ to evaluate the elastic properties of the superlattice composed of TO1289/ $\mathrm{SC}_{12}$ gold nanoclusters. The bulk, shear, and Young's moduli of the superlattice are shown in Figs. 10(a), 10(b) and 10(c), respectively. As a direct check of these results, we have performed also constant pressure simulations at a number of temperatures in which the superlattice was equilibrated at several pressures in increments of $10 \mathrm{MPa}$. The compressibility is obtained using $\Delta V / V=X \Delta P(\Delta P<0)$ where $\Delta V$ is the change in volume, and the bulk modulus is given by $B=1 / X$. It is evident from Fig. 10a that the results are consistent with those obtained from the strain fluctuations method. As a point of reference, we also applied this direct method, using constant temperature simulations, to estimate the bulk modulus of liquid dodecane at selected temperatures. It is interesting to note that when the temperature exceeds the phase transition point, $T_{0}$, the bulk moduli of the superlattice and liquid dodecane are essentially equal. For temperatures below $T_{0}$ the bulk dodecane freezes into a structure exhibiting both ordered and disordered domains, having a bulk modulus less than that of the superlattice.

A quantity that is commonly used to characterize the elastic behavior of a material is the shear modulus, relating strain and applied stress for a shear in two dimensions. We show in Fig. 10b the shear modulus $G_{12}$ and the average of $G_{13}$ and $G_{23}$. As the temperature decreases below $T_{0}$, the moduli rise almost linearly until $T \sim 100 \mathrm{~K}$ and then they increase slowly to values typical of plastics (at $300 \mathrm{~K}$, the shear modulus of polystyrene is $\sim 1.33 \mathrm{GPa}^{48}$ ). When the temperature reaches and exceeds $T_{0}$ the manner by which the moduli vary undergoes an abrupt change, and it exhibits a thermal dependence which is very similar to the Young moduli (see Fig. 10c), with values typical of elastomers. 


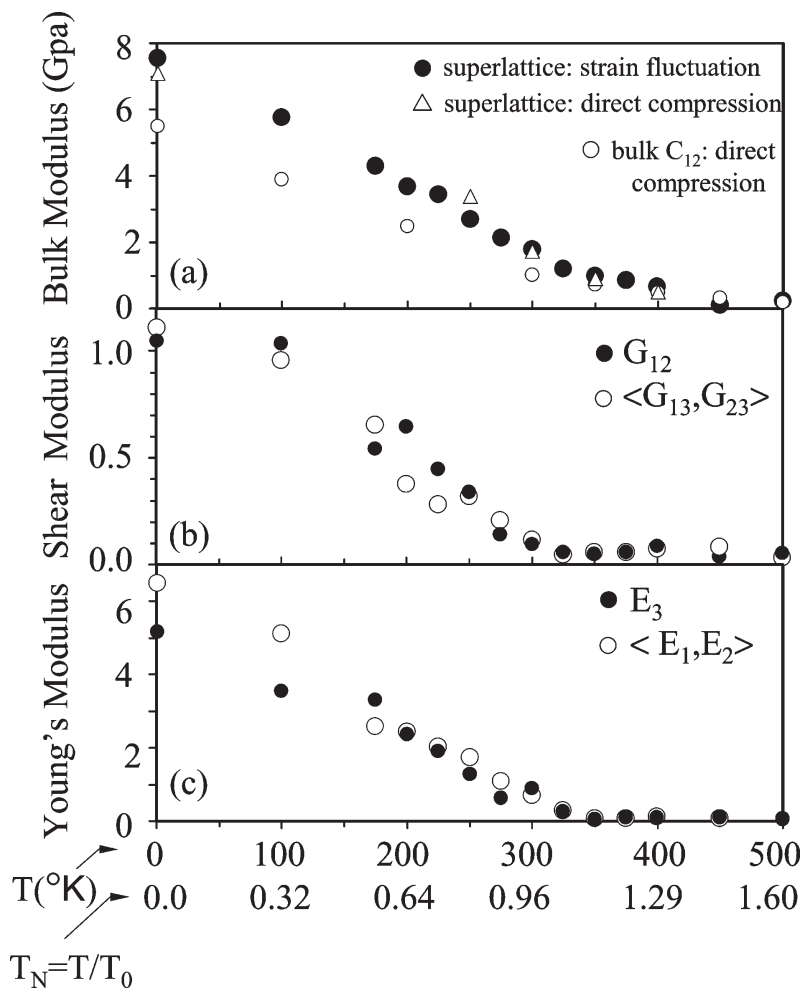

Fig. 10 Elastic constants of the simulated superlattices described in Figs. 8 and 9. In (a) we show results for the bulk modulus, obtained through the use of the strain-fluctuation formalism as well as via direct compression. Results obtained from MD simulations of bulk dodecane are also included. At the bottom axis we give both the actual temperature, as well as the temperature normalized to the transition (melting) temperature of the material.

The Young modulus $E_{3}$ and the average $\left\langle E_{1}, E_{2}\right\rangle$ of the 1,2 axes are shown in Fig. 10c. One observes that these moduli increase almost linearly for lower temperatures. At lower temperatures the moduli are of the order of several GPa and they are comparable to those of plastics (at $300 \mathrm{~K}$ the Young modulus of polystyrene is $\sim 3.6 \mathrm{GPa}^{48}$ ), but much lower than that of glass (at $300 \mathrm{~K}$ the Young modulus of pyrex glass and fused silica are $62 \mathrm{GPa}$ and $73 \mathrm{GPa}$, respectively ${ }^{48}$ ). When the temperature exceeds the transition point the Young modulus falls to lower values rather abruptly; compare to the behavior of the bulk modulus, see Fig. 10a, the percent of trans conformation shown in Fig. 9a, and the physical dimensions of the computation cell displayed in Fig. 9b. It eventually reaches values in the range 0.05 to $0.1 \mathrm{GPa}$, that are typical of certain elastomers; at 300 $\mathrm{K}$ the Young modulus of flexible polyurethane is $\sim 0.045 \mathrm{Gpa}$ and that of polyisoprene is in the range $^{49}$ of 0.002 to $0.1 \mathrm{GPa}$.

These comparisons were performed at $\sim 300 \mathrm{~K}$. Another way of comparing the behavior of different materials consists of plotting the elastic moduli versus the normalized temperature $T_{\mathrm{N}}=T / T_{0}$ (see bottom of Fig. 10), where the transition temperature $T_{0}$ for this study was found to be $\sim 312 \mathrm{~K}$ (see Fig. 8). The behavior of the Young moduli parallels that of typical elastomers and some epoxies (a cross-linked polymer), ${ }^{49}$ where below $T_{\mathrm{N}}=1$ the Young modulus increases very rapidly to values of $\sim 5 \mathrm{GPa}$ as the material enters a glassy regime (at which point the increase slows down). For $\mathrm{T}_{\mathrm{N}}$ slightly above unity the materials are in a rubbery regime with Young's moduli on the order of $\sim 0.8 \mathrm{MPa}$ (elastomer) and $\sim 0.3 \mathrm{Gpa}$ (epoxy).

In closing this section we remark that while there has been some experimental research on the freezing and melting transitions of passivating chain molecules, essentially no published experimental work addresses the elastic properties of superlattices built from passivated nanocrystals. 


\subsection{Free energy of formation}

Insights into the driving forces for assembly of nanoparticle arrays may be obtained from considerations of their formation free-energy; indeed, certain studies of the free energy of twodimensional films of passivated nanocrystals, ${ }^{50,51}$ as well as of three-dimensional superlattices, ${ }^{4,52,53}$ have appeared. In order to study in some detail the relationships between the total energy and the free energy of formation of a superlattice (including entropic contributions), we construct first a bcc superlattice (made of $\mathrm{Au} \mathrm{TO} 1289 / \mathrm{SC}_{12}$ gold nanocrystals at $375 \mathrm{~K}$ ) with a lattice constant large enough so that the clusters are initially outside of each other's interaction range. Then the lattice constant is decreased in steps, with lengthy equilibrations at each stage, until a broad range of lattice constants has been explored. At each stage the passivated cores are constrained to reside on bcc unit cell sites but are otherwise unconstrained. Subsequently, the free energy of the superlattice as a function of the lattice constant (or the core-core nearest-neighbor distance), as well as the entropic contribution to the free energy, can be evaluated with the use of the calculated changes in the pressure, volume and internal energy relative to the initial isolated-cluster stage, and in conjunction with standard thermodynamic relations. As will be shown below, the entropic contribution is relatively large and it is comparable to that of the potential energy.

At each of the constant volume equilbration stages we calculate the relative Helmholtz free energy, $F=U_{\text {rel }}-T S$, where $U_{\text {rel }}$ is the energy of the system relative to that when the passivated cores are (initially) well separated, and $S$ is the entropy of the system. From the differential relation $\left.\partial_{V} F\right|_{T}=P$ (see ref. 54; note that a positive increment of volume in this reference corresponds to a negative change in volume as used here) one ascertains that when the pressure of the system passes through the equilibrium value $(P=0)$, the free energy $(F)$ reaches an extremum (minimum in this case). For the isothermal conditions of this study, one has that $F={ }_{\infty} \int^{R} P \mathrm{~d} V$; i.e. the change in free-energy occurring in the course of assembly of the superlattice, equals the reversible work done in going from the reference state (where the passivated clusters are well-separated, denoted as $\infty$ in the integral) to the final superlattice state where the clusters are separated by a distance $R$. From the MD simulations we can determine directly both $U_{\text {rel }}$ and $P$ for a number of bec lattice constants. Using a spline fit to these points we perform the above integration to compute the free energy and then, using $S=-\left(F-U_{\text {rel }}\right) / T$, we deduce the entropic contribution to the free energy (for an earlier formulation of this method of evaluation of the entropy contribution to the free energy, see ref. 55). The variables discussed above are plotted in Fig. 11a as a function of the corecore nearest-neighbor distance $R_{\mathrm{nn}}$. For $U_{\mathrm{rel}}$ and $P$, the continuous lines are the result of spline fits (the discrete equilibration points are indicated by symbols for $U_{\text {rel }}$ ), while for the free energy and entropy the continuous lines are deduced from $U_{\text {rel }}$ and $P$ as described above. We observe that, as expected, the pressure passes through zero at the minimum of the free energy $F$ and that this minimum occurs at a lattice constant quiet different than that associated with the minimum of the relative energy $U_{\text {rel }}$. Most importantly, the entropic contribution to the free energy is found to be as large as the relative potential energy of the superlattice.

We turn next to a brief discussion of the various contributions to the relative energy $U_{\text {rel }}$. In Fig. $11 \mathrm{~b}$ we display the two largest contributions, along with the relative and free energies. The dominant contributions to the energy are from the chain-chain van der Waals (vdW) interactions, with those associated with interactions between chains passivating different gold cores accounting for almost the entire relative energy $U_{\text {rel }}$. Other contributions amount at most to a mere $0.5 \%$ of the total relative energy. It is important to note that the smallest contribution is from the $\mathrm{vdW}$ interactions between the metal cores. This finding, that agrees with our earlier work, ${ }^{30}$ contradicts certain model assumptions used previously by other researchers. ${ }^{53,56}$

\subsection{Pressure-induced structural transformations}

A relatively unexplored area in studies of superlattices made of passivated clusters pertains to the properties of superlattices that are subjected to an externally applied pressure, namely their compressibility and pressure-volume equation of state (EOS). Some research and modeling efforts in this general direction focused on two-dimensional (2D) nanocluster assemblies, for the purposes of elucidating the phase diagrams of such systems, construction of pressure versus area relationships, understanding of thermal and optical properties, and explorations of electronic transport 


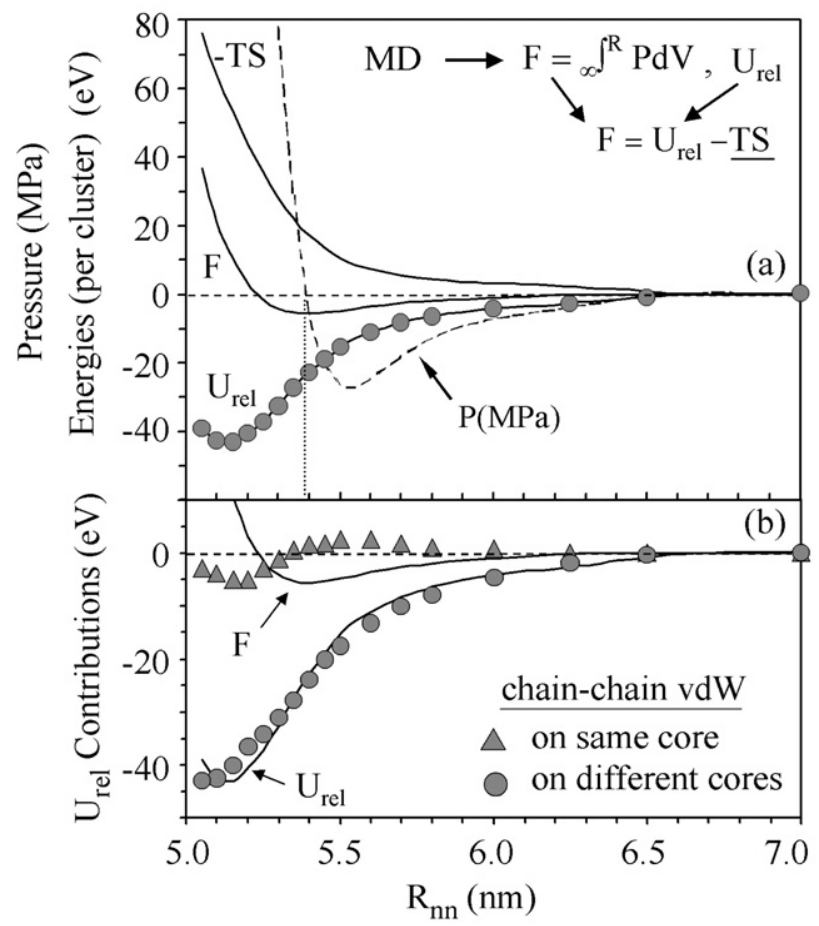

Fig. 11 Results from MD simulations pertaining to the formation of the superlattice made of gold TO1289/ $\mathrm{SC}_{12}$ nanoclusters. In (a) we show the pressure $(P$, dashed line), and the entropic term $(-T S)$, the energy of the assembled system relative to the isolated clusters $\left(U_{\text {rel }}\right)$, and the total Helmholtz free energy $(F)$. Contributions to $U_{\text {rel }}$ from van der Waals interactions between molecular chains belonging to the same core nanocluster, or between chains corresponding to different cores are shown in (b).

processes between the metal cores. ${ }^{36,37,57}$ Aiming at developing an EOS for 3D supperlattices, we have started with a superlattice made of $\mathrm{TO} 1289 / \mathrm{SC}_{4}$ gold nanoclusters equilibrated initially at $\mathrm{P}=0$, and have gradually increased the external pressure on it; the pressure was applied incrementally, such that after each pressure increase the system was allowed to equilibrate prior to further pressurization. As we discuss below this process resulted in a pressure- induced structural phase transformation from a tetragonal $\mathrm{fcc}(\mathrm{tfcc})$ superlattice structure to one with a body-center tetragonal (bct) symmetry.

While the construction of the EOS will be discussed in detail elsewhere, we present in the following the results of our aforementioned pressurization simulation. At zero applied pressure the symmetry of the superlattice (described above) was tfcc with a ratio $(c / a)_{\text {conv }} \sim 0.92$ (between the long and shorter axes of the conventional fcc cubic four-atom unit cell); note that the unit cell displayed in Fig. 12 is not the conventional one (unless noted otherwise the use of $(a, c)$ will refer to the unit cell shown in Fig. 12). When the pressure was increased from 100 to $200 \mathrm{MPa}$, a distinct change in the superlattice symmetry occurred as it transformed from tfcc to bct. The pressureinduced structural transformation was detected by us through examination of the unit-cell vectors, as well as through analysis of the powder X-ray scattering intensity ${ }^{58}$ calculated during the pressurization process (see Fig. 12). The transformation involved a decrease of the angle between the $a$-axes of the tfcc (non-conventional) unit cell (starting from $84^{\circ}$ at $P=100 \mathrm{MPa}$ and transforming to $79^{\circ}$ at $P=200 \mathrm{MPa}$ ) and an abrupt change in the length of the $c$-axis (see Fig. 12), resulting at $P=200 \mathrm{Mpa}$ in a (non-conventional) two-cluster unit cell which we find to be of the bct type; for pressures of $200 \mathrm{MPa}, 500 \mathrm{MPa}, 1 \mathrm{Gpa}$ and $3 \mathrm{Gpa}$, the bet superlattice had $(c / a)$ ratios of 1.12 , $1.09,1.07$ and 1.07 , respectively.

Other materials that undergo pressure-induced structural transformations include $\mathrm{C}_{60}$ (see ref. 59), $\mathrm{Rb}_{3} \mathrm{C}_{60}$ (see ref. 60) and $\mathrm{BeS}$ (see ref. 61), and some of these are particularly relevant to our 


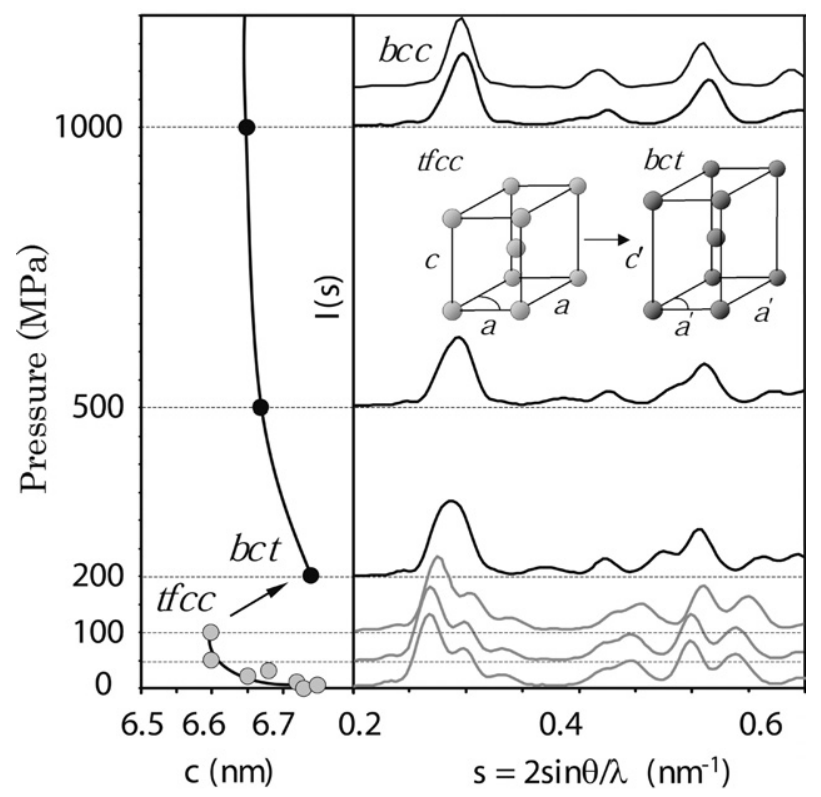

Fig. 12 Results from MD simulations of pressure-induced transformations. The superlattice that has been simulated results from the assembly of gold nanoclusters with 1289 atoms, passivated by a monolayer of butane thiols at $300 \mathrm{~K}$. On the left we show the variation of the $c$-axis (the longest axis of the unit cell) as a function of the applied hydrostatic pressure. The initial structure of the superlattice is tetragonal fcc (tfcc), and for $P>100$ $\mathrm{MPa}$ it structurally transforms, assuming a bet structure at $200 \mathrm{MPa}$. Further increase of the pressure results (at sufficiently high pressure) in an approximate bcc structure. On the right we show calculated powder X-ray intensities corresponding to the structures predicted at various applied pressures, showing an evolution from a tfcc to a bct, and ultimately to an approximate bcc superlattice structure. The non-primitive unit cells illustrate the tfcc-to-bct transformation. For the initial (at $P=0$ ) tfcc unit cell $(c / a)=1.5$ and the angle between the axes at the base of the unit cell is $83^{\circ}$. For the unit cell of the bct structure at $P=200 \mathrm{MPa}\left(c^{\prime} / a^{\prime}\right)=1.57$ and the base-angle is $79^{\circ}$. For a perfect bcc unit cell $(c / a)=1.63$ and the base-angle is $70.5^{\circ}$.

work. Underlying the particular interest and challenge of developing an EOS for superlattices formed through the assembly of passivated nanocrystallites, is the fact that they are composed of relatively hard (essentially incompressible) cores (made of gold nanoclusters in this study), which are separated from each other by softer, more compressible, material (i.e. the passivating alkythiol chains). Because of this unique composition, when pressure is applied it is only the distance between the core surfaces that is reduced while the dimensions of the solid cores remain unaffected. Consequently, standard EOS formalisms may not accurately describe such a system as there is a large portion of the volume that is incompressible. ${ }^{59,60}$ Instead, in an attempt to describe the results of our simulations (see Fig. 12), and for the purpose of developing an EOS for the system, we have adopted an approach that has yielded satisfactory results in other investigations; ${ }^{59-61}$ in this approach we use a modification of the Birch $\operatorname{EOS}^{62}$ that was originally derived for the description of the compressibility of cubic crystals. A comprehensive discussion of this topic is outside the limits of this article and it will be given elsewhere.

\section{Summary}

Materials particles of nanometer dimensions, in dispersions or in agglomerated forms (e.g., as powders, thin films, or superlattice assemblies) are of increasing interest due to the size-dependent specificity and selectivity of their physical characteristics, conferred to them by their finite size. Indeed, investigations of size-evolutionary patterns of materials properties (e.g., energetics, structure, thermodynamics, spectral, and chemical reactivity) are a principal theme in modern cluster and materials science. 
In this article we have explored, with the use of extensive molecular dynamics (MD) simulations, several principal issues pertaining to the energetics of formation of superlattices made through the assembly of passivated nanoclusters, the interactions that underlie the cohesion of such superlattices, and the unique mechanical, thermal and structural properties that they exhibit. Our investigations focused on crystalline gold nanoclusters of variable size (with radii of $0.83 \mathrm{~nm}$ to 1.75 $\mathrm{nm}$, corresponding to cluster compositions ranging from about 100 to over 1000 gold atoms) passivated by monolayers of alkylthiol molecule of variable chain-length (up to dodecane thiol), and assembled into superlattices of various structural motifs. Our main findings may be summarized as follows:

(i) Through systematic investigations we have formulated an analytic model (the optimal packing model, OPM) that correlates in a unified manner several structural characteristics of superlattice assemblies. We have shown that a number of properties that serve to specify structural characteristics of superlattice assemblies, as well as the organization of the nanocluster buildingblocks of which such assemblies are comprised, may be achieved through the use of a parameter, $x$, that expresses the ratio between the extended molecular chain-length of the passivant and the radius of the solid nanocluster. This parameter, in conjunction with a postulated approximate equality (justified a posteriori by the apparent success of the model) between the volume occupied by a fully extended passivating molecule and the volume occupied by a passivating molecule in the superlattice assembly, allowed us to organize and systematize a large amount of available experimental data (see Fig. 4), as well as our simulation results (see Fig. 5). Furthermore, the OPM allows to predict the phase-boundary between superlattice crystallographic symmetries corresponding to different structural motifs, that is, the model gives a value $x_{\mathrm{B}}$ below which $\mathrm{fcc} / \mathrm{hcp}$ superlattice structures are found, and above which more open structures, bcc/bct-type, emerge.

(ii) Superlattices formed through the assembly of solid nanocrystallites passivated by organic monolayers, are unique materials where the space between the solid cores is largely occupied by relatively soft organic matter. Such superlattices were found here, through the use of MD simulations in conjunction with a strain fluctuation method, to exhibit remarkable mechanical and thermo-mechanical response characteristics.

Room temperature MD simulations of the mechanical response of superlattice assemblies revealed characteristic evolution of the elastic moduli of these materials as a function of $x$. We predict that above the phase-boundary value $x_{\mathrm{B}}$ (that is, when open bcc/bct structures of the superlattice are found) the behavior of the bulk modulus resembles that of short-chain (of the order of 10 carbons) alkane liquids at room temperature, while the shear and Young's moduli behave like those of elastomers. Superlattices comprised of passivated nanocrystals with values below $x_{\mathrm{B}}$ are predicted to exhibit mechanical response like solid alkanes, plastics or elastomers, depending on the value of $x$ (Figs. 6 and 7).

Our predictions with regard to the elastic moduli and their temperature dependence (see Fig. 10), may be summarized as follows: at higher temperature, that is above the melting temperature of the passivating molecular chains, the bulk modulus of the superlattice material would be similar to that of a simple alkane liquid, and the Young and shear moduli will exhibit values similar to elastomers. At temperatures below the above-mentioned melting point, when states of the superlattice exhibiting ordered chain arrangements (such as chain-bundles) develop, the bulk modulus and it's temperature dependence would be similar to those of alkane crystals, while the Young and shear moduli will exhibit behaviors typical of plastics. These predictions provide insights into existing experimental results, as well as motivate new basic research into the physical properties of these novel types of materials, and their potential technological applications.

(iii) Structural transformations of passivated nanocluster superlattices may be induced thermally (see Fig. 9) or through the application of external pressure (see Fig. 12). With regard to the latter, we note that the pressure-induced tfcc-to-bct structural transformation discussed by us in section 2.5 and displayed in Fig. 12, can be related to the chain-packing considerations discussed in the context of the OPM (see point (1) above) as follows: when the superlattice has initially (i.e. at zero pressure) a fcc-type structure (where the chains do not fill all of the available space), the application of hydrostatic compression may result (at some critical pressure) in occupation of the empty available space in the superlattice, thus driving a transformation to a stabilized bcc-type structure. This behavior suggests a certain analogy between the fcc-to-bcc evolution caused by chain-filling of the free volume as the parameter $x$ is varied, and the compression-driven transformation. 
Such externally-induced structural transformations, that are accompanied by significant variations in mechanical characteristics (as well as other physical properties, such as electronic transport), are of fundamental and potential technological interest.

(iv) The entropic contribution to the formation free energy of the superlattice (via assembly of the passivated cluster building-blocks) is large and it equals in magnitude the potential energy part. The major contribution to the cohesive potential energy of the superlattice derives from van der Waals interactions between molecules passivating neighboring nanoclusters.

\section{Appendix}

To perform large-scale MD simulations in an efficient manner we make use of the fact that the internal $(\mathrm{Au}-\mathrm{Au})$ interactions in the nanocrystalline gold core are of no particular significance for the material properties that we explore. Consequently, we treat a gold core as a solid body using quaternion dynamics. ${ }^{63}$ In this method the alkylthiol molecules interact with core atoms and the resulting forces are translated into center-of-mass and angular forces that are then integrated to advance the core's position and orientation. The interaction between metallic gold cores (through a dodecane medium) was modeled via an appropriate expressions for the van der Waals forces; ${ }^{56,64,65}$ the Hamaker constant ${ }^{64}$ for gold-gold attraction through dodecane is $\approx 2 \mathrm{eV}$.

The equations of motion derive from a Lagrangian formalism in which the interactions discussed above are incorporated though the corresponding potential functions. ${ }^{41,66}$ Included in this approach are terms that allow both the shape and volume of a simulated lattice to vary dynamically enabling the system to explore crystallographic symmetry changes and phase transitions (i.e. melting transitions).

The intra- and inter-molecular interactions for the alkylthiol molecules, $\mathrm{S}\left(\mathrm{CH}_{2}\right)_{n} \mathrm{CH}_{3}$, were modeled through united-atom interaction potentials, which include bond-stretching, bond-angle, dihedral-angle, and non-bonded interactions, and the interactions between the alkyl $\left(\mathrm{CH}_{3}\right.$ and $\mathrm{CH}_{2}$ ) segments and the gold core atoms were described via potentials fitted to experimentally measured desorption energies. The sulfur-gold interaction was modeled through the use of a Morse potential fitted to experimental binding energies, bond distances and vibrational force constants of sulfur adsorbed on $\mathrm{Au}(111)$ and $\mathrm{Au}(100)$ surfaces (see ref. 30 for a more detailed discussion of the above and related references).

The preparation of a passivated nanocrystal involves controlled adsorption and desorption of gas phase alkylthiols (see refs. 30 and 31 for details). These passivated cores can then be rotated to any desired initial orientation and placed at the positions of the desired superlattice unit cell. The lattice constant is initially large enough that there is no interaction between the molecules on different cores and constrained constant pressure simulations are performed with a constant-shape unit cell, with only the alkythiol molecules treated dynamically. Temperature control, typically to $300 \mathrm{~K}$, is achieved via stochastic thermalization; that is using a Maxwell-Boltzmann distribution corresponding to the desired temperature to select the velocities assigned to a randomly selected set of particles at stochastically chosen times. The pressure (set initially at $\sim 100 \mathrm{Mpa}$ ) is reduced gradually thus allowing the system to assume a compact superlattice configuration. At this point the effective velocities describing the rate of change of the unit cell dimensions ${ }^{41}$ are zeroed and the system is allowed to equilibrate at a small pressure $(\sim 10 \mathrm{Mpa})$, and subsequently the velocities are zeroed again and the system equilibrated under zero pressure. Finally, the full dynamics of the calculation cell (allowing shape and size variations of the unit cell), as well as the solid-body dynamics (allowing rotation and translation of the passivated cores) are allowed, and further equilibration at constant temperature (controlled now through velocity scaling) and pressure is performed. The system is then ready as a starting point for further studies.

The time-step used in integrating the equations of motion was $3 \mathrm{fs}$. Equilibration times for passivated cluster superlattices can sometimes be quite large $\left(10-20 \mathrm{~ns} \sim 3-7 \times 10^{6}\right.$ integration steps) in order to allow adequate time for the possible structural and/or phase transitions. ${ }^{30,31}$ The size of a simulated system depends on the number of ligands (passivating molecules) per nanocrystalline core, and on the number of cores in a unit cell. For a two-cluster superlattice unit cell, composed of Au TO1289 cores (each of which having 258 passivating dodecane-thiol molecules) there are 6708 molecular segments and 2578 gold atoms). 


\section{Acknowledgements}

We thank Dr J. Gao for useful discussions pertaining to evaluation of the free energy, and acknowledge the assistance of Dr B. Yoon in preparing the figures. This research has been supported by a grant from the US Department of Energy.

\section{References}

1 G. M. Whitesides and B. Crzybowski, Science, 2002, 295, 2418.

2 G. M. Whitesides and M. Boncheva, Proc. Nat. Acad. Sci. USA, 2002, 99, 4769.

3 J.-M. Lehn, Proc. Nat. Acad. Sci. USA, 2002, 99, 4763.

4 R. L. Whetten, M. N. Shafigullin, J. T. Khoury, T. G. Schaaff, L. Vezmar, M. M. Alvarez and A. Wilkinson, Acc. Chem. Res., 1999, 32, 397.

5 C. P. Collier, T. Vossmeyer and J. R. Heath, Annu. Rev. Phys. Chem., 1998, 49, 371.

6 M. Brust and C. J. Kiely, Colloids Surf. A, 2002, 202, 175.

7 A. C. Templeton, W. P. Wuelfing and R. W. Murray, Acc. Chem. Res., 2000, 33, 27.

8 W. M. Tolles, Mater. Res. Soc. Bull., 2000, (October), 36.

9 P. Moriarty, Rep. Prog. Phys., 2001, 64, 297.

10 A. Ulman, An Introduction to Ultrathin Organic Films, Academic Press, San Diego, CA, 1991.

11 A. Ulman, Chem. Rev., 1996, 96, 1533, and references therein.

12 L. H. Dubois and R. G. Nuzzo, Ann. Rev. Phys. Chem., 1992, 43, 437.

13 R. G. Nuzzo and D. L. Allara, J. Am. Chem. Soc., 1983, 105, 4481.

14 See list of references in ref. 1 of R. H. Terrill, et al.,, J. Am. Chem. Soc., 1995, 117, 12537.

15 P. Fenter, A. Eberhardt and P. Eisenberger, Science, 1994, 266, 1216, and references therein.

16 M. Brust, M. Walker, D. Bethell, D. J. Schriffin and R. J. Whyman, J. Chem. Soc. Chem. Commun., 1994, 801.

17 M. Brust, D. Bethell, D. J. Schriffin and C. J. Kiely, Adv. Mater., 1995, 7, 795.

18 R. L. Whetten, J. T. Khoury, M. Alvarez, S. Murthy, I. Vezmar, Z. L. Wang, P. W. Stephens, C. L. Cleveland, W. D. Luedtke and U. Landman, Adv. Mater., 1996, 8, 428.

19 R. L. Whetten, J. T. Khoury, M. M. Alvarez, S. Murthy, I. Vezmar, Z. L. Wang, C. L. Cleveland, W. D. Luedtke and U. Landman, in Chemical Physics of Fullerenes 5 and 10 Years Later, ed. W. Andreoni, Kluwer, Dordrecht, 1996.

20 G. T. Schaaff, M. Shafigulin, J. T. Khoury, I. Vezmar, R. L. Whetten, W. G. Cullen, P. N. First, W. C. Gutierrez, J. Ascensio and M. J. Jose Yacaman, J. Phys. Chem., 1997, 101, 7886.

21 R. H. Terrill, et al., J. Am. Chem. Soc., 1995, 117, 12537.

22 P. C. O’Hara, D. V. Leff, J. R. Heath and W. M. Gelbart, Phys. Rev. Lett., 1995, 75, 3466.

23 M. Dorogi, J. Gomez, R. Osifchin, R. P. Andres and R. Reifenberger, Phys. Rev. B, 1995, 52, 9071.

24 A. Badia, S. Singh, L. Demers, L. Caccia, G. R. Brown and R. B. Lennox, Chem. Eur. J., 1996, 2, 359.

25 A. Badia, L. Cuccia, L. Demers, F. Morin and R. B. Lennox, J. Am. Chem. Soc., 1997, 119, 2682.

26 A. Badia, W. Gao, S. Singh, L. Demers, L. Cuccia and L. Reven, Langmuir, 1996, 12, 1262.

27 J. Hautman and M. L. Klein, J. Chem. Phys., 1989, 91, 4994.

28 J. Hautman and M. L. Klein, J. Chem. Phys., 1990, 93, 7483.

29 W. Mar and M. L. Klein, Langmuir, 1994, 10, 188, and references therein.

30 W. D. Luedtke and U. Landman, J. Phys. Chem., 1996, 100, 13323.

31 W. D. Luedtke and U. Landman, J. Phys. Chem. B, 1998, 102, 6566.

32 H. Hakkinen, R. N. Barnett and U. Landman, Phys. Rev. Lett., 1999, 82, 3264.

33 M. N. Shafigullin, PhD Thesis, 1999, Georgia Institute of Technology, Atlanta, GA.

34 G. A. McConnell, A. P. Gast, J. S. Huang and S. D. Smith, Phys. Rev. Lett., 1993, 71, 2102.

35 G. A. McConnell and A. P. Gast, Phys. Rev. E, 1996, 54, 5447.

36 J. R. Heath, M. Knobler and D. V. Leff, J. Chem. Phys B., 1997, 101, 189.

37 W. M. Gelbart, R. P. Sear, J. R. Heath and S. Chaney, Faraday Discuss., 1999, 112, 299.

38 W. D. Luedtke and U. Landman, unpublished results.

39 M. Parrinello and A. Rahman, J. Chem. Phys., 1982, 76, 2662.

40 M. Sprik, R. W. Impey and M. L. Klein, Phys. Rev. B, 1984, 29, 4368.

41 M. Parrinello and A. Rahman, Phys. Rev. Lett., 1980, 45, 1196.

42 J. R. Ray, M. C. Moody and A. Rahman, Phys. Rev. B., 1985, 32, 733.

43 J. R. Ray and A. Rahman, J. Chem. Phys., 1984, 80, 4423.

44 CRC Handbook of Chemistry and Physics, ed. R. C. Weast, CRC Press, Boca Raton, FL, 55th edn., 1974.

45 N. Sandhyarani, M. R. Resmi, R. Unnikrishnan, K. Vidyasagar, M. Shuguang, M. P. Antony, G. P. Selvam, V. Visalakshi, N. Chandrakumar, K. Pandian, Y. Tao and T. Pradeep, Chem. Mater., 2000, 12, 104.

46 N. Sandhyarani, M. P. Antony, G. P. Selvam and T. Pradeep, J. Chem. Phys., 2000, 113, 9794.

47 N. Sandhyarani, T. Pradeep, J. Chakrabarti, M. Yousuf and H. K. Sahu, Phys. Rev. B, 2000,62, R739.

48 W. P. Mason, American Institute of Physics Handbook, McGraw-Hill, New York, 3rd edn., 1972. 
49 L. J. Gibson and M. F. Ashby, Cellular solids: structure and properties, Cambridge University Press, New York, 1997.

50 P. C. Ohara and W. M. Gelbart, Langmuir, 1998, 14, 3418.

51 P. C. Ohara, J. R. Heath and W. M. Gelbart, Angew. Chem. Int. Ed. Engl., 1997, 36, 1078.

52 B. A. Korgel and D. Fitzmaurice, Phys. Rev. B, 1999, 59, 14191.

53 B. A. Korgel, S. Fullam, S. Connolly and D. Fitzmaurice, J. Phys. Chem B, 1998, 102, 8379.

54 C. Kittel and H. Kroemer, Thermal Physics, W. H. Freeman and Company, San Francisco, 1980.

55 J. Gao, W. D. Luedtke and U. Landman, J. Phys. Chem. B, 1997, 101, 4013.

56 P. C. Ohara, D. V. Leff, J. R. Heath and W. M. Gelbart, Phys. Rev. Lett., 1995, 75, 3466.

57 G. Markovich, C. P. Collier and J. R. Heath, Phys. Rev. Lett., 1998, 80, 3807.

58 A. Guinier, X-ray Diffraction, W.H. Freeman and Company, San Francisco, 1963.

59 H. A. Ludwig, W. H. Fietz, F. W. Hornung, K. Grube, B. Wagner and G. J. Burkhart, Z. Phys. B, 1994, 96, 179.

60 H. A. Ludwig, W. H. Fietz, F. W. Hornung, K. Grube, B. Renker and G. J. Burkhart, Physica C, 1994 , 234, 45.

61 C. Narayana, V. J. Nesamony and A. L. Ruoff, Phys. Rev. B, 1997, 56, 14338.

62 F. Birch, Phys. Rev., 1947, 71, 809.

63 M. P. Allen and D. J. Tildesley, Computer Simulation of Liquids, Oxford University Press, New York, 1987.

64 H. C. Hamaker, Physica, 1937, 4, 1058.

65 D. Bargeman and F. Van Voorst, J. Electroanal Chem., 1972, 37, 45.

66 C. L. Cleveland, J. Chem. Phys., 1988, 89, 4987. 